\title{
MODELLING AND SIMULATION OF THE PHARMACY OF A NEW HOSPITAL
}

\author{
Michel GOURGAND, Fateh MEBREK, Alain TANGUY \\ LIMOS, CNRS UMR 6158, Université Blaise Pascal, \\ Complexe des Cézeaux, 63173 Aubière Cedex, France \\ E-mail: gourgand@isima.fr, mebrek@isima.fr, tanguy@isima.fr
}

\section{KEYWORDS}

Pharmacy service management, ARIS modelling, Modelling environment, SIMULA, WITNESS.

\begin{abstract}
Pharmacy is one of the services providing the hospital. Its mission concerns the supply of the drugs for the patients which remain in the hospital. This paper describes the modelling methodology used to specify a knowledge model of the pharmacy service of a new French hospital. We first describe the pharmacy service of the old hospital using ARIS analysis tool, then we define the functioning of this service in the new hospital and test several hypothesis by the way of simulation models using the SIMULA language and the simulation tool WITNESS.
\end{abstract}

\section{INTRODUCTION}

The organization or the reorganization of the logistic chain constitutes a major stake for the public health establishments, because its improvement is one of the principal reserves of savings in this sector. It is indeed obvious that the budgetary profits must in priority being realized on the invisible elements in the broad sense for the patient (Benanteur et al. 2000). In this direction, the optimization of the pharmaceutical logistic chain is very important: from the pharmaceutical product arrival to the patient beds (Baboli et al. 2003). The rational organization of the pharmacy (Aarons et al. 2001) may be regarded as a necessary evolution. However, major difficulties may appear during this evolution, because the hospital pharmacy has a great diversity of stored products.

Within the same pharmacy, the system must be able to manage several areas dedicated to different kinds of storage: an area can be intended for the articles to store at low temperature (cold room), another one for dangerous products (safe room)... This division in areas can also be relevant according to the rate of inventory turnover of certain articles. Indeed, the frequency of restocking varies in an important way with a care unit to the other one. The articles with weaker rotation will be generally located at a less accessible place by the personnel.
The organization of the arrangement of the pharmaceutical products and their installation must satisfy a certain number of aims such as the accessibility to the stored articles, the improvement of the working conditions, the reduction in time and the errors of preparation. These objectives involve the definition of criteria of internal organization of pharmacy (Chow and Heaver 1994) such as the availability of the products, the management policy, the cost of storage, ergonomics as well as the constraints to be respected such as the climatic conditions of the buildings and available surfaces. For each site, we can specify a certain number of parameters such as the length, the width and the height of the stored references, the mass and the family of products (sterile material, medical devices and objects of bandages), or conditionings (limps, boxes, pallets, etc.).

Within the framework of the changing of the pharmacy area of the old hospital, we studied several organization scenarios of stocks and restocking. This organization should make it possible to provide pharmaceutical products under optimum conditions of quality and safety. The assignment of the various buildings to the drugs and the medical devices will have to respect the regulation of hospital pharmacy. Various technological constraints and activity priorities (emergencies, easy circulation, simplification of internal transport and delivery...) will have also to be taken into account.

This paper describes the pharmacy service management then it details the proposed modelling methodology, used to design simulation models, finally it presents experiments and concluding remarks.

\section{PHARMACY SERVICE MANAGEMENT}

The drug circuit (Madic 2001) starts with the regulation and continues with the dispensation, that includes the pharmaceutical analysis and the delivery of drugs. It is completed by the distribution to the patient and it includes the management of the drugs: laboratory orders, inventory control, out-of-date, withdrawal of batches (in the pharmacy as in the cupboards of the services), choice of the available drugs at the hospital and constitution of the booklet of the drugs. 
We can classically do graph the drug life cycle and the actor actions, as follows:

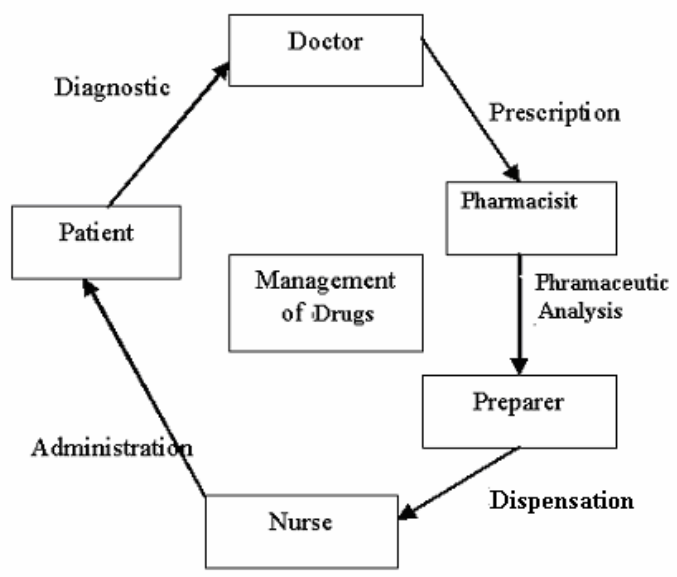

Figure 1: Drug Circuit

However, the pharmacy in the new hospital will be centralized on the main hospital site, also located in the same city, which will provide all the drugs and the devices needed by the services and the care units, as shown in figure 2.

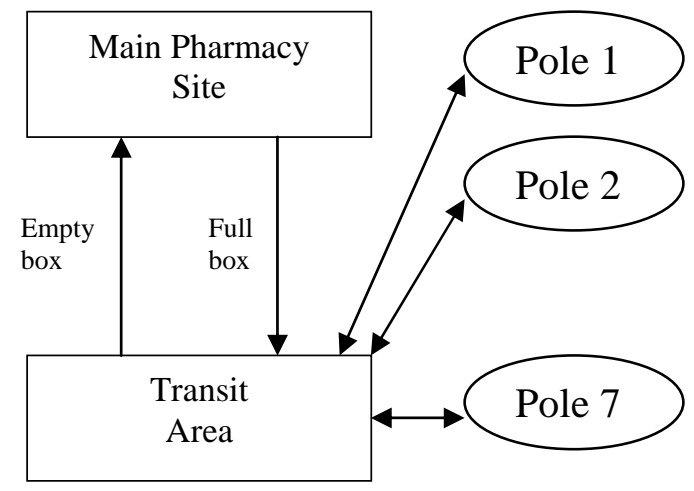

Figure 2: Pharmacy Flow

\section{MODELLING METHODOLOGY}

The modelling approach ASDI (Analysis Specification Design Implementation), we present, was first proposed by Gourgand and Kellert (Gourgand and Kellert 1991) for production systems. It is based on the construction of two classes of models: the knowledge model and action models. The knowledge model describes the structure and the operation of the system in a natural or graphic language; it is built using the three subsystems (logical, physical and decisional). For an existing system, the knowledge model contains the knowledge acquired by the system observation. For a system to be designed, the knowledge model contains the specifications of the future system. An action model is a translation of the knowledge model in a mathematical formalism or a programming language, for example a simulation language, allowing the evaluation of the selected performance criteria. Several action models can be built starting from the same knowledge model. The consecutive construction and the use of these two models constitute the modelling process.

\section{Modelling Environment}

The main aim of the modelling methodology consists in building a knowledge model as generic as possible that enables the implementation of action models for specific systems of the domain. The knowledge model remains an open model that is enhanced by each study of hospital systems. The knowledge management and the implementation of action models implies the computer aid provided by a modelling environment that could be open in order to include new and accurate methods or tools.

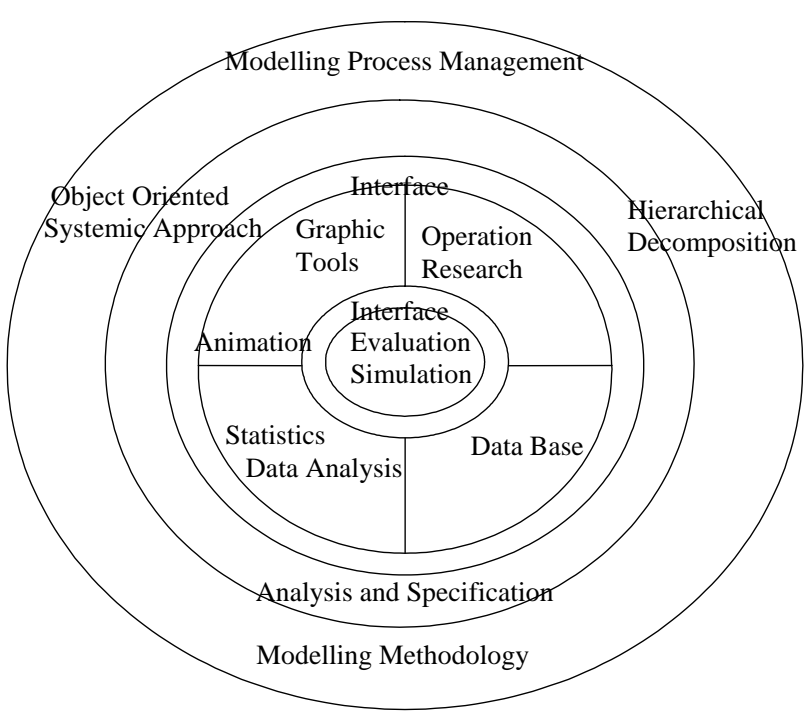

Figure 3: Modelling Environment

The modelling environment (figure 3) helps the user to exchange information with different partners of the project and facilitates the design and the implementation of action models during the phase of information extraction from the knowledge model. It is an attempt to the automation of the modelling process using knowledge formalization, data analysis, characteristic computation, operational research, evaluation, graphic and animation tools.

The first knowledge model of the hospital logistic system operations and structure is formalized by means of the software tool ARIS (Architecture of Integrated Information Systems), suggested by Scheer (Scheer 2002). This tool is suitable to describe organizations, processes and activities (Gourgand et al. 2005), as well as entity relationship models (Chen 1976). Some parts of the hospital system are specified with the UML language. An extra simulation module is available for ARIS, but for graphical needs, financial, accuracy and 
policy reasons WITNESS simulation tool was preferred so as to design and to implement action models. Let us notice that the information extraction from ARIS files is not so easy that we may expect, and let us remark that WITNESS was specifically designed for industrial systems.

\section{Modelling Process}

The iterative modelling process (figure 4) was first introduced in (Gourgand and Kellert 1991), (Green and Roseman 2000). It is composed of four steps:

- Construction of a knowledge model based on the analysis and the specification of the system,

- Development of an action model, using the knowledge model,

- Exploitation of an action model,

- Modifications or actions on the system.

The construction of the knowledge model must be carried out in collaboration with the experts of the system domain. This model must remain coherent in time, whatever its level of smoothness and the evolutions brought to the system at the time of its use. The objectives must be clearly identified in order to know the problems to solve. The construction of the knowledge model consists in collecting and formalizing the knowledge on the studied system. A functional and structural analysis formalizes the system in a written form. The specification must enable the modelling experts and the system experts to agree about the operations of the system.

The modelling methodology is mainly based on the definition of the knowledge model specification composed of the description of three subsystems and their interactions:

- The physical subsystem (PSS) consists in the physical entities providing a service or participating in an operation, the PSS topology defines the position of physical devices and their connections,

- The logical subsystem (LSS) contains the transactions treated by the system and the definition of services and elementary operations in regard to the treatment of transaction flows and depending on the entities in the system,

- The decisional subsystem (DSS) contains the management policies, resource allocation and system functioning rules.

During the phase of functional and structural analysis, a method of decomposition can be employed to facilitate the formalization of knowledge when the studied system is complex. A phase of specification allows the expression of the operation of the entities of the system and flows. Specifically, the system of control must be specified in a precise way, taking into account the events and the synchronization of the actions. The format of the input data must be specified. The specification must allow the experts in modelling and the experts of the system to agree on the operations (real or wished) of the system. The construction of the knowledge model requires the use of adapted tools and/or formalization. The knowledge model of a system is formalization in a natural or graphic language of the structure and of the operations of this system.

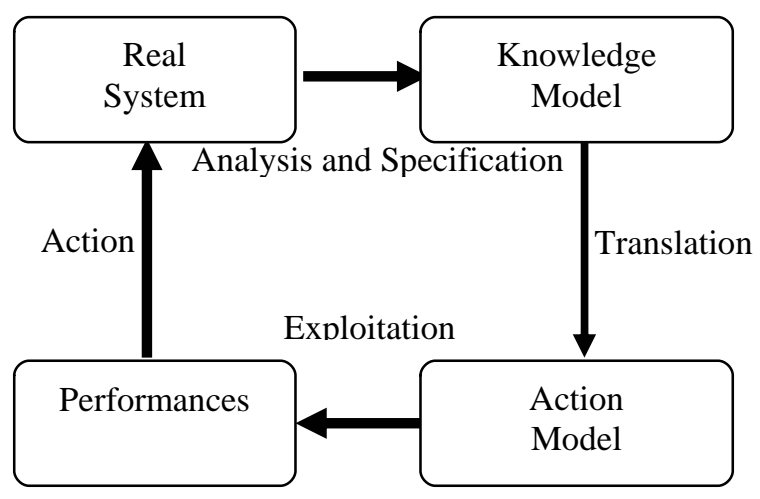

Figure 4: Modelling Process

We used the ARIS tool to describe and specify the pharmacy service. To be able to use ARIS in order to design the knowledge model, several assumptions of modelling are to be taken into account.

- Each activity (function in ARIS) is attached to one or more organizational units of the hospital system (a care unit, the operating theatre suite, pharmacy, etc.);

- Each event posses its own information document, it is used by several processes and it is reference in one or more documents of the information system (medical file of the patient, file of the operating theatre suite, etc.);

- The reference documents (ARIS models) describe the key processes. Taking into account our modelling objectives, we retained, in the ARIS toolbox, two types of representation in order to detail the processes and the relations between services.

The processes are studied using an Event-driven Chain of Process (ECP) that shows the processes have a clearly definite structure and the ECP will describe flows in the logical subsystem. The relations within the services and between services are represented by a business flow chart for the decisional subsystem.

\section{ARIS Modelling}

The Event-driven Chains of Process represent the flow chart and the functioning of an organization. They describe the relations between the objects concerning the data, the organizational functions and consequently they represent the processes. The sequence of functions of a process is represented by chains of processes. In these chains, it is possible to indicate arrivals and the starting event for each function. The events start the functions and they are generated by other events.

The graphic symbol of the event is a hexagon. The function is symbolized by a rectangle (figure 5). 


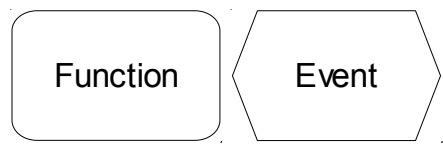

Figure 5: Function and Event

As the events define the state or the condition which starts a function as well as the state which marks its completion, the starting and the arrivals of such an ECP are always events. An event can start several functions simultaneously and, conversely, a function can generate several events. To be able to represent these ramifications and these loops of treatment in an $\mathrm{ECP}$, it is possible to use logical connectors (And, Or, Xor...) in the shape of circle.

The human and material resources are detailed in figure 6 . They may directly generate flows depending on their status of dynamic (able to move) or static resources. Some static human resources are able to generate specific flows such as information flows. The logistic agents are dynamic resources able to carry material dynamic resources. The hospital pharmacy is not only concerned by drugs but by a lot of products and devices, for instance: sterile medical products (CAMS) or non sterile furniture (CAL).

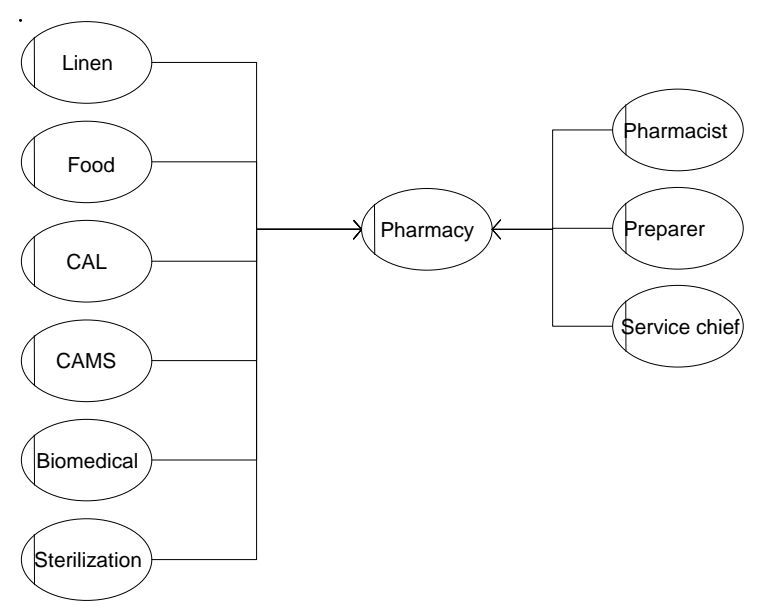

Figure 6: Pharmacy flows

The main pharmacy site provides drugs by trucks in the morning. The drugs are temporally stored at the transit area and then distributed to the care units. Figure 7 shows the specification of the service pharmacy by an ECP. After the truck arrival, the drugs are delivered at the care units of the new hospital. There are 39 care units structured on 7 poles, and 11 agents work at the transit area.

\section{SIMULATION MODELS}

The proposed action models are based on queueing networks because the customer are represented by products, boxes or patients and they are processed by active resources such as agents or nurses who need passive resources such as carts or medical devices. This formalism is suitable to study waiting durations in a stochastic context. The direct evaluation is not easy to implement due to passive resource management, special probability distributions and transient behaviour. So we designed simulation models of the pharmacy of the new hospital using the SIMULA language and the WITNESS tool.

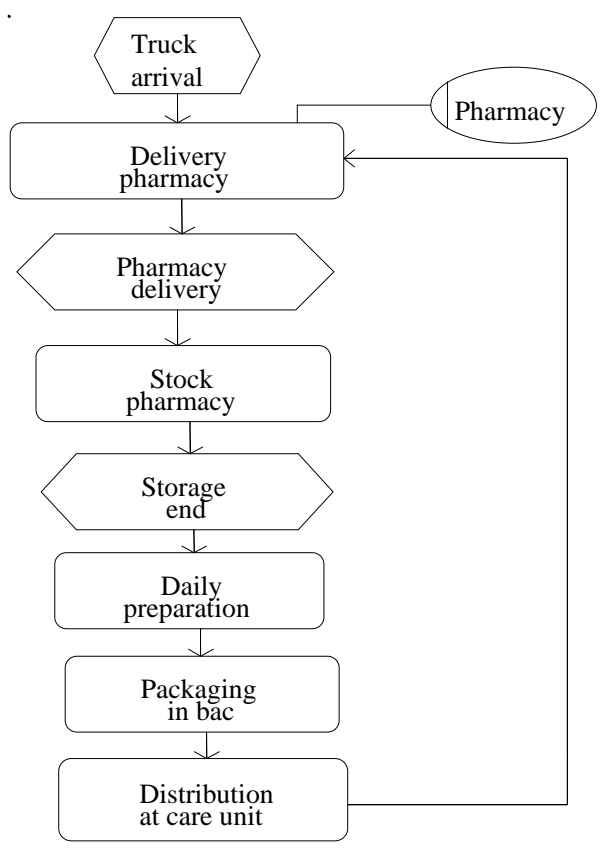

Figure 7: ECP Pharmacy

\section{Queueing Network Model}

Figure 8 describes a queueing network model that represents an action model. In our case study we consider the pharmacy with its entities: human resources (personnel) and material (storage areas of the pharmacy, carts, carriage...).

This model is very simple but enables to estimate the resource utilization rates, the mission and request waiting times; to test hypothesis consistency concerning empty travel times or to tune parameters. Of course, a steady state or transient behaviour study can be foreseen. In table 1 we presented the different probability distributions used to simulate the models of pharmacy. The treatment is composed of the following tasks: material loading, travelling, nurse availability, material unloading and return.

The uniform probability distribution is often regarded as the worst distribution, but it possesses great qualities: a compact support, simple computation, model verification, and its convolution with other uniform distributions generates trapezoid, triangular and more sophisticated distributions. So, face to the lack of consistent data for the studied system, this choice is not worse than another one, and it is very 
easy to change the probability distribution in the realized models.

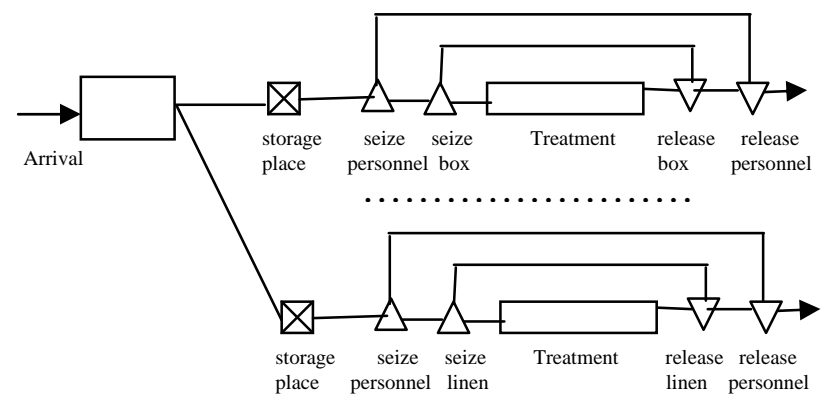

Figure 8: Queueing Network Model

Table 1: Experimental Data

\begin{tabular}{|l|l|}
\hline Delays & Distributions \\
\hline Nurse availability & Uniform $(0,2)$ \\
\hline Travelling & Uniform $(5,10)$ \\
\hline Material loading & Uniform $(10,20)$ \\
\hline Return & Uniform $(6,11)$ \\
\hline Material unloading & Uniform $(5,10)$ \\
\hline
\end{tabular}

We assume that the truck arrival follows a uniform $(0$, 60) distribution. An exponential arrival is not exactly justified and there is only one truck arrival during half a day (7:00 to $14: 30)$. We consider that the simulation starts at 0 (7:00) and finishes at 480 minutes (14:00) a 30 minutes pause has to be added to time greater than 240 (11:00). The truck unloading is supposed uniform $(5,10)$. So, the 9 drug lots are available on the transit area at the mean time 37.5 and the maximum time 70 . The 4 carts limit the number of parallel travels for instance with 2, 3, 4 and 5 agents: $(2,2,2,2,1)$, (3, $3,3),(4,4,1)$ and $(4,4,1)$. The availability of the drug lots at the poles may be a critical condition. We can estimate a mean time $37.5+3 * 39.5=156(9: 36)$ for 3 and more agents. Other performance criteria could be estimated in this way. Beside the availability of the drugs, the utilization rate of the agents may be considered. Anyway, the system functioning is less efficient for 2 or only one agent. Of course, it is possible to test various scenarios, different characteristics and loads for the poles, using the realized simulation models.

\section{SIMULA Model}

The SIMULA language has proved its capacity to implement different kinds of simulation models. It includes coroutines and simulation processes with discrete events. The Gpsss class provides the basic objects such as the single service (facility), storage, the concepts of transaction and statistical region, moreover one simulation report is automatically generated. It can thus be employed as in GPSS programming but with all the capacities of an object oriented simulation language.
The designed models were realized using a transaction orientation and a process approach. Among several hypotheses, we assume that the transactions are drug lots. They are concurrently unloaded from the truck and stored on the transit area, then agents carry them to the poles using one of four special carts. The storage object fills three roles: multiple servers, stock and set of resources; thus, a storage is able to stand for an agent or a cart. The stochastic duration of a treatment is realized by a call to the hold method. The statistics concerning waiting or treatment durations are collected by a region object. The methods enter_object and leave_object are called to manipulate the basic objects of the Gpsss class.

The used objects and the results, for 100 replications with a $1 \%$ risk, are represented in table 2 . The maximum and mean drug availability dates are given by D.AvailMax and D.Avail; then the mean durations per drug lot for the storage on transit area $\mathrm{T}$.StoreTZ, each distribution mission T.Mission and waiting for a cart WT. Cart; finally the utilization duration of each agent UT. Agent and the duration he waits for a cart WT.Cart/Ag. A criterion is not reported when it does not evolve. The drug availability date decreases from 396.8 to the constant value 152.7 for 4 and more agents. Of course, there are no cart waiting for 4 or less agents and it is close to 20 for more agents. As expected, the agent utilization time decreases from 358.5 to 52.8 .

Table 2: Results of SIMULA Model

\begin{tabular}{|c|c|c|c|}
\hline 1 Agent (s) & D.Avai & $\operatorname{Max}$ & 50 \\
\hline D.Avail & 396.808 & +- & 5.868 \\
\hline T.StoreTz & 157.221 & +- & 1.667 \\
\hline T.Mission & 39.833 & +- & 0.331 \\
\hline WT. Cart & 0.000 & +- & 0.000 \\
\hline UT. Agent & 358.495 & +- & 2.983 \\
\hline WT.Cart/Ag & 0.000 & +- & 0.000 \\
\hline & & & \\
\hline 3 Agent (s) & D.Avail & 1 Max & 199.015 \\
\hline D.Avail & 163.717 & +- & 5.080 \\
\hline T.StoreTz & 38.319 & +- & 0.468 \\
\hline T.Mission & 39.833 & +- & 0.331 \\
\hline UT.Agent & 119.498 & +- & 0.995 \\
\hline 4 Agent (s) & D.Avail & 1 Max & 193.741 \\
\hline D.Avail & 152.687 & +- & 5.036 \\
\hline T.StoreTz & 24.680 & +- & 0.320 \\
\hline T.Mission & 39.833 & +- & 0.331 \\
\hline $\begin{array}{l}\text { UT.Agent } \\
5 \text { Agent (s) }\end{array}$ & 89.624 & +- & 0.746 \\
\hline WT.Cart & 8.035 & +- & 0.111 \\
\hline UT.Agent & 86.162 & +- & 0.757 \\
\hline WT.Cart/Ag & 14.463 & +- & 0.201 \\
\hline 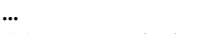 & & & \\
\hline 11 Agent (s) & & & \\
\hline WT.Cart & 24.680 & +- & 0.320 \\
\hline UT.Agent & 52.783 & +- & 0.498 \\
\hline WT.Cart/Ag & 20.192 & +- & 0.262 \\
\hline
\end{tabular}

\section{WITNESS Model}

To pass from the knowledge model to the WITNESS simulation model, the following principle is used: the medical drugs and devices are represented by articles 
entering in stock, then we represent the agents by resources and the care units by machines. The processing times are defined by the probability distributions given in table 1.

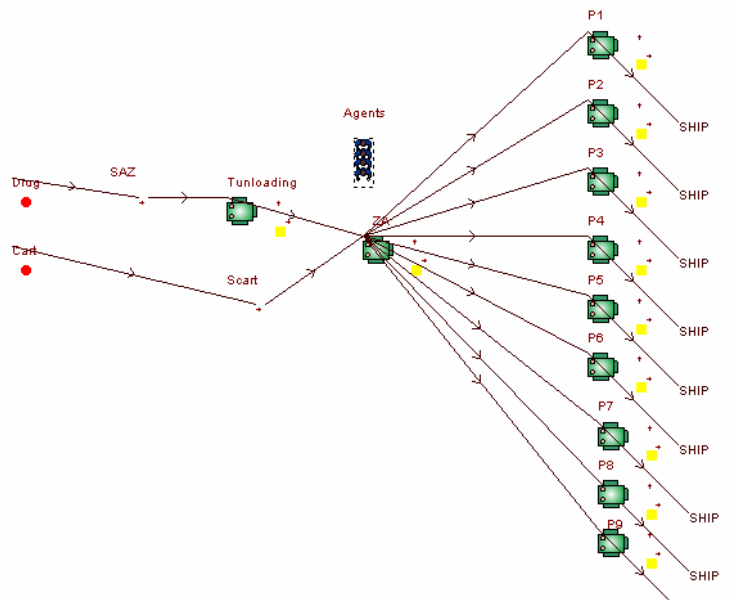

Figure 9: WITNESS Model

The simulation model is staged by three steps shown in figure 9 as it is depicted in figure 8: in the first step the truck is coming from central hospital between 7 and 8 in the morning. In the second step drugs (Drugs) are stored in the transit area (ZA) after unloading by agents (Agents). Finally the agents share nine missions in the seven poles. The mission scenario is described as follows: an agent arrives with his pharmacy cart, loads drugs, and distributes drugs in the seven poles. The mission is achieved when the agent returns with his empty pharmacy cart. We can find single or double missions for each pole. We model double missions by means of two virtual poles instead of only one pole.

The work load of the agents is less easy to obtain from the simulation results because they are formatted for an industrial system. Furthermore, only one simulation is executed, we have to manually change the seed and run a next one, thus no automation of replications. So, we simulate the model for 4 to 11 agents and each time we look at the given utilization rate of the agents to estimate the work duration of the agents. The model results were compared with models realized with a new version of the Queueing Network Analysis Package QNAP2 (Potier 1983) for validation purpose.

\section{CONCLUSION}

We presented the pharmacy service and the utility of the knowledge model specified by means of the ARIS tool, as well as the passage from this model towards an action or simulation model realized in SIMULA or with WITNESS. It is not easy to extract information from ARIS files. Moreover, complex systems need specification tools for the synchronization of its processes (Petri networks...) but the ARIS ECP did not appear enough fine for this aim. It was not possible for us to test the specific ARIS simulation module.
The decision-making aid tool, we realized with the Gpsss class and SIMULA, provides results with confidence intervals on the utilization rate of the pharmacy resources. WITNESS enables the use of a graphic interface with animation of the missions to each pole. SIMULA and QNAP2 are more suitable to provide and to validate, automatically generated models including replication and optimization processes for Windows and UNIX operating systems.

In prospect the models will be improved by connecting several services which have a relationship to the pharmacy and hospital logistic. Of course, a data and a statistics collection must be deeper realized so as to propose actions on the studied system.

\section{REFERENCES}

Aarons L.; I. Nestorov; G. Graham; S. Duffull; L. Aarons; E. Fuseau and P. Coates. 2001. "Modeling and simulation for clinical trial design involving a categorical response: A phase II case study with naratriptan". Publication Pharm.Res. Vol 18, 1210-1219.

Baboli A.; A. Guinet; T. Hassan; M.T. Brandon; B. Charpiat and G. Leboucher. 2003. "Identification des flux de la logistique pharmaceutique d'un hôpital public". GISEH, Lyon, 17-18 janvier. 392.

Benanteur Y., R. Rollinger, J-L. Saillour. 2000. "L'organisation logistique et technique à l'hôpital". Éditions ENSP, 185.

Chen P. 1976. "The entity relationship model - Toward a unified view of data". ACM Transaction on data base system. Vol $1, N^{\circ} 1$ mars.

Chow G. and T.D. Heaver. 1994. "Logistics in the Canadian Health Care Industry". Canadian Logistics Journal, vol. 1, $n^{\circ}$ 1. 29-73.

Gourgand M. and P. Kellert. 1991. "Conception d'un environnement de modélisation des systèmes de production". 3ème congrès international de Génie Industriel, Tours.

Gourgand M.; F. Mebrek and A. Tanguy. 2005. "Hospital logistic modelling and simulation case study: brancardage". ESM05, Porto, Portugal, 2426 october. $372-377$.

Green P. and M. Roseman. 2000. "Modelling: An ontological evaluation". In Information systems, ated process, vol 25.

Madic A. 2001. "Informatisation du circuit du médicament à l'hôpital : de l'intention à la réalisation". Thèse Ph. D en pharmacie. Université d'Angers octobre. 183.

Potier D. 1983. "New user's introduction to QNAP2". INRIA Report $n^{\circ} 40$, October. 123.

Scheer A.W. 2002. "ARIS - Business Process Modelling”. Springer. 